\title{
Microgrid Operational Optimization with Dynamic Voltage Security Constraints
}

\author{
Agnes M. Nakiganda \\ Dept. of Electronic and Electrical Eng \\ University of Leeds, UK \\ el14amn@leeds.ac.uk
}

\author{
Thierry Van Cutsem \\ Fund for Scientific Research (FNRS) \\ University of Liege, Belgium \\ t.vancutsem@ulg.ac.be
}

\author{
Petros Aristidou \\ Dept. of Elec \& Comp Eng. \& Informatics \\ Cyprus University of Technology, Cyprus \\ petros.aristidou@cut.ac.cy
}

\begin{abstract}
Emergency islanding of a microgrid (MG) can be vital in ensuring continuity of power supply to a local network during major contingencies in the main grid. However, such an event will inadvertently cause large voltage transients except in the trivial case where zero power is exchanged. The under/over voltages can result in the disconnection of the local distributed generators within the MG, increasing the risk of a local blackout. In this paper, we present a MG operational optimization strategy that includes dynamic voltage constraints aiming at enhancing MG resilience during emergency situations. A dynamic optimization technique, based on sequential constraint transcription, is used to formulate the dynamic voltage security constraints applied to the steady-state problem in a computationally efficient manner. An iterative approach ensures that the dynamic constraints are updated in relation to the optimized operating point of the MG. The performance of the proposed approach is investigated on a 30-bus medium-voltage MG by considering a potential disconnection at each hour of the day, subject to daily load and generation profiles.
\end{abstract}

Index Terms-Dynamic grid support, dynamic optimization, emergency islanding, fault ride-through, microgrid, operational planning.

\section{INTRODUCTION}

In recent years, the frequency of catastrophic events affecting power systems such as extreme weather events, natural disasters and major faults has been on the rise. This necessitates the design of resilient infrastructure to enhance the survivability and resilience of the grid when subject to such stressful conditions. One key such solution to enhance power systems' resilience is to split the network into selfsufficient islands that can continue to ensure the provision of critical services [1]. Microgrids (MGs) are flexible systems consisting of distributed energy resources (DERs) able to operate both autonomously and in connection to the bulk grid. These systems have been proposed as such a solution to ensure power system resilience and continuity of supply during emergency events [2].

The nature of high impact and low probability catastrophic of events, means that a MG might be required to abruptly disconnect from the bulk network and form an island that should both survive the islanding transients, as well as ensure supply continuity in the post-islanded state. Violations of the secure

This work was supported by the Engineering and Physical Sciences Research Council (EPSRC) in the UK under grant reference EP/R030243/1.

978-1-6654-3597-0/21/\$31.00 @2021 IEEE operating limits of the DERs in both the transient and postislanded steady-state of the MG will lead to the activation of their protective devices, disconnecting them from the network and increasing the risk of cascaded failures. Moreover, the ever increasing ratio of Converter-Based Generators (CBGs) to Synchronous Generators (SGs) in MGs results in radical changes both in the dynamic and operational characteristics of the system [3].

Previous studies on the dynamic performance of MGs subsequent to unintentional islanding events have shown large excursions in both frequency and voltage during transient periods for a fault-triggered islanding condition as compared to planned islanding events [4], [5]. In this paper, we focus only on the voltage response of the MG during emergency islanding conditions. To improve the dynamic performance of voltage during faults, grid codes often require DERs to stay connected to the network by implementing Fault-Ride Through (FRT) capabilities, and where possible to provide reactive power support by implementing Dynamic Voltage Support (DVS) [6], [7]. The impact of these capabilities on DERs has been widely studied [8]-[10], indicating transient performance enhancement in all cases. However, this benefit is dependent on the DERs remaining connected during and after the transients and their protective devices not being activated. In the event of emergency islanding, the disconnection of the distributed generators in the MG will affect the survivability of the MG further impeding its ability to support the critical local load therefore endangering its reliability and resilience.

Existing literature on optimizing voltage security focuses on bulk transmission systems and looks into either long-term voltage stability margins [11]-[13] or the post fault transient voltage recovery [14], [15] - neglecting the effect of FRT limitations i.e. low-voltage ride-through (LVRT) and highvoltage ride-through (HVRT) security limits on the generating units. Moreover, the effect of dynamic voltage security during MG operational optimization has not been adequately studied. Finally, past studies often neglect the effect of active power on the voltage trajectory, this can be crucial especially in mediumand low-voltage systems with high $R / X$ ratio.

In this study, we enhance a MG operational planning problem to account for constraints related to the dynamic behaviour of the MG voltages during unintentional islanding at each hour of the planning horizon. This way, the pre-fault operating 


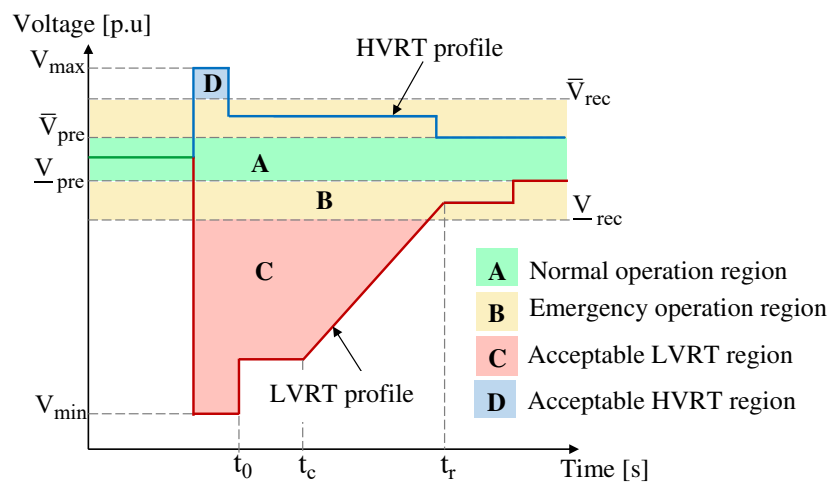

Fig. 1. Voltage FRT profile for DERs with fault occuring at time $t_{0}$.

point is appropriately modified to ensure the system's ability to survive the post-fault transients and attain a stable response in case of the MG emergency islanding. To achieve this, we include equations for the voltage evolution during the FRT and dynamic post-fault response of the MG into the operational planning problem, and apply path constraints to ensure that voltage security is maintained in the post-islanded state.

The contribution of this paper is therefore two-fold:

1) We propose an iterative algorithm that uses time-domain analysis to formulate dynamic voltage security constraints transcribed into the operational optimization problem to enhance system security and survivability in the case of emergency islanding.

2) We investigate the effect of the scheduled operating point, considering both active and reactive power, on the transient voltage performance of the MG. We concurrently study the effect on the generator FRT protections, as well as the post-fault recovery transient voltages to ensure performance within the grid code limits.

The remainder of the paper is structured as follows: Section II presents the dynamic voltage security criterion and voltage support capabilities adopted for the DERs while Section III outlines the optimization problem setup and solution methodology adopted. We present the case study and discussion in Section IV and provide concluding remarks in Section V.

\section{Voltage Security CRiterion}

During major contingencies, such as unintentional MG islanding, the DER FRT ability ensures that power generation is not lost while DVS reduces the level of voltage dips reducing the risk of system blackout. However, if the voltage exceeds the technical limit, the unit is tripped to avoid damage. This action can result in further disconnections of DERs and a risk of cascading failures.

\section{A. FRT Security Criterion}

The FRT requirement include thresholds on the LVRT, the HVRT, and the transient post fault recovery voltage. These requirements are set within a pre-defined voltage versus time profile that varies based on the grid standards and protection requirements. Fig. 1 illustrates the FRT curve that requires generators to remain connected to the grid given that voltages remain within the reference curves. The normal and emergency operation region have security upper/lower bounds defined by $\underline{V}_{\text {pre }} / \bar{V}_{\text {pre }}$ and $\underline{V}_{\text {rec }} / \bar{V}_{\text {rec }}$, respectively, while maximum LVRT and HVRT levels are denoted by $V_{\min }$ and $V_{\max }$.

\section{B. DVS Capability of CBGs}

Voltage response in generators is normally subject to discrete controls based on the operation region as illustrated in Fig. 1. Additionally, to prevent the risk of cascading failures, grid codes have been updated with requirements of FRT and grid-support by DERs. SG units have adjusted over-/underexcitation limiters to ensure momentary reactive power support during over/under voltage conditions. However, such controls are not readily available in CBGs. Control strategies detailed in [10], [16] present models that implement the DVS capabilities of CBGs. Therein, to ensure efficient support, the CBG will prioritise reactive power output to active power in the event that voltages drop lower or rise higher the normal operating region (see Fig. 1). The variation in injected reactive current, $i_{Q}(t)$, with respect to the measured voltage, $V(t)$, can be modeled as:

$$
i_{Q}(t)=I_{\mathrm{Q}, \text { pre }}+ \begin{cases}K_{\mathrm{i}}\left(\underline{V}_{\text {pre }}-V(t)\right) & \text { if } V(t)<\underline{V}_{\text {pre }} \\ K_{\mathrm{a}}\left(\bar{V}_{\text {pre }}-V(t)\right) & \text { if } V(t)>\bar{V}_{\text {pre }} \\ 0 & \text { otherwise }\end{cases}
$$

where $I_{\mathrm{Q}, \text { pre }}$ is the pre-fault reactive current generated by the CBG and parameters $K_{\mathrm{i}}$ and $K_{\mathrm{a}}$ define the respective rate of change of reactive current with respect to a change in voltage. The functionality of the model is detailed in [16].

It should be noted that the maximum available reactive power reserve of the generators will be limited by the current ratings of the CBG and subject to the dynamic security limits on the voltage at the generator connection node.

\section{Problem Formulation}

In this section, we present the proposed formulation of the MG optimization problem. A multi-period optimization problem is formulated to minimize the grid-connected operational cost, with extra dynamic constraints to ensure the survavibility of the MG in the case of unintentional islanding at each hour. The solution approach involves the integration of the differential and algebraic equations that describe the dynamic response of the system into the operational planning problem through the adoption of a computationally efficient technique.

\section{A. Optimization Model}

The objective and constraints of the problem to ensure steady-state and dynamic security are defined as follows:

1) Objective: The objective function in grid connected mode minimises the operational costs of the DERs as well as the cost of power exchange with the grid. It is defined as:

$$
\begin{aligned}
& \min \sum_{h \in \mathcal{H}}\left(\left(c^{\mathrm{b}_{\mathrm{P}}} \cdot P_{h}^{\mathrm{b}}-c^{\mathrm{s}_{\mathrm{P}}} \cdot P_{h}^{\mathrm{s}}\right)+\left(c^{\mathrm{b}_{\mathrm{Q}}} \cdot Q_{h}^{\mathrm{b}}-c^{\mathrm{s}_{\mathrm{Q}}} \cdot Q_{h}^{\mathrm{s}}\right)\right) \\
& +\sum_{h \in \mathcal{H}} \sum_{g \in\{\mathcal{R}, \mathcal{C}\}}\left(c_{g}^{\mathrm{P}} \cdot P_{g h}+c_{g}^{\mathrm{Q}} \cdot Q_{g h}\right)+\sum_{h \in \mathcal{H}} \sum_{n \in \mathcal{N}}\left(c_{n}^{\mathrm{l}_{\mathrm{v}}} \cdot P_{n h}^{\mathrm{l}_{\mathrm{v}}}\right)
\end{aligned}
$$


where, for each hour $h$ contained in set $\mathcal{H}, P_{h}^{\mathrm{b}} / Q_{h}^{\mathrm{b}}$ is the active/reactive power imported from the grid at costs $c^{\mathrm{b}_{\mathrm{P}}} / c^{\mathrm{b}_{\mathrm{Q}}}$, while $P_{h}^{\mathrm{s}} / Q_{h}^{\mathrm{s}}$ is the power exported at costs $c^{\mathrm{SP}} / c^{\mathrm{sQ}}$. Each generator $g \in\{\mathcal{R}, \mathcal{C}\}$ where $\mathcal{R}$ and $\mathcal{C}$ is a set of SGs and CBGs respectively, has operational costs for generated active/reactive power $P_{g h} / Q_{g h}$ indicated by $c_{g}^{\mathrm{P}}$ and $c_{g}^{\mathrm{Q}}$, respectively. We consider demand response capabilities in the MG by allowing for the flexible part of load, $P_{n h}^{\mathrm{l}_{\mathrm{v}}}$, to be shifted subject to a penalty denoted by $c_{n}^{l_{\mathrm{v}}}$ where $n$ is the node index contained in set of all nodes $\mathcal{N}$.

2) Power Flow Constraints: At each node $n \in \mathcal{N}, n^{\mathrm{u}} / n^{\mathrm{d}}$ denoting the nodes upstream/downstream of $n$, the power flow equations, considering a radial network topology are formulated using the DistFlow model [17] as:

$$
\begin{array}{ll}
S_{n^{\mathrm{u}} n h}+S_{h \mid n=\mathrm{pcc}}^{\mathrm{b}}-S_{h \mid n=\mathrm{pcc}}^{\mathrm{s}}+\sum_{g \in\{\mathcal{R}, \mathcal{C}\}} S_{g h} & \\
\quad=\sum_{n^{\mathrm{d}} \in \mathcal{N}^{\mathrm{d}}} S_{n n^{\mathrm{d}} h}+S_{n h}^{\mathrm{l}}+z_{n^{\mathrm{u}} n} \cdot l_{n^{\mathrm{u}} n h}, & \forall n, h \\
v_{n^{\mathrm{u}} h}-v_{n h}=2 \cdot \Re\left(z_{n^{\mathrm{u}} n}^{*} \cdot S_{n^{\mathrm{u}} n h}\right)+\left|z_{n^{\mathrm{u}} n}\right|^{2}, & \forall n, h \\
v_{n^{\mathrm{u}} h} \cdot l_{n^{\mathrm{u}} n h}=\left|S_{n^{\mathrm{u}} n h}\right|^{2}, & \forall n, h
\end{array}
$$

where $S_{n^{\mathrm{u}} n h}$ indicates the apparent power flows in the line connecting nodes $n$ and $n^{\mathrm{u}}, l_{n^{\mathrm{u}} n h}$ the square of line current, $z_{n^{\mathrm{u}} n h}$ the line impedance and $\mathcal{N}^{\mathrm{d}}$ the set of nodes connected downstream to $n$. Variable $v_{n^{\mathrm{u}} h}$ is the square of node voltage, while $S_{h \mid n=\text { pcc }}^{\{\mathrm{b}, \mathrm{s}\}}, S_{g h}$ and $S_{n h}^{\mathrm{l}}$ denote the apparent power: exchanged with the bulk grid at the point-of-common-coupling (PCC); generated by DERs; and the load, respectively. Constraint (3c) is non-convex and is relaxed using second-order cone programming into an inequality as $l_{n^{\mathrm{u}} n h} \geq\left|S_{n^{\mathrm{u}} n h}\right|^{2}$.

3) Grid Exchange and Load Constraints: The lower/upper bounds of variables are denoted by $\bullet / \bar{\bullet}$. Power bought/sold from/to the grid is limited as:

$$
\begin{array}{lll}
0 \leq P_{h}^{\mathrm{b}} \leq \bar{P}_{h}^{\mathrm{b}} \cdot y_{h}^{\mathrm{P}}, & 0 \leq P_{h}^{\mathrm{s}} \leq \bar{P}_{h}^{\mathrm{s}} \cdot\left(1-y_{h}^{\mathrm{P}}\right), & \forall h \\
0 \leq Q_{h}^{\mathrm{b}} \leq \bar{Q}_{h}^{\mathrm{b}} \cdot y_{h}^{\mathrm{Q}}, & 0 \leq Q_{h}^{\mathrm{s}} \leq \bar{Q}_{h}^{\mathrm{s}} \cdot\left(1-y_{h}^{\mathrm{Q}}\right), \quad \forall h
\end{array}
$$

where variables $y_{h}^{\mathrm{P}} / y_{h}^{\mathrm{Q}}$ prevent the simultaneous import and export of active/reactive power. The hourly load consumption is subject to the load profiles as:

$$
\begin{aligned}
0 \leq P_{n h}^{1} & \leq \bar{P}_{n h}^{1}, \quad 0 \leq Q_{n h}^{1} \leq \bar{Q}_{n h}^{1}, & & \forall n, h \\
{[P / Q]_{n h}^{1} } & =[P / Q]_{n h}^{l_{\mathrm{f}}}+[P / Q]_{n h}^{\mathrm{v}_{\mathrm{v}}}, & & \forall n, h \\
\sum_{h \in \mathcal{H}} P_{n h}^{\mathrm{l}} & =E_{n}, & & \forall n
\end{aligned}
$$

where subscripts $\mathrm{f}$ and $\mathrm{v}$ represent the fixed and variable part of the load. Constraint (4e) ensures that the energy consumption of the load $E_{n}$ for the day is maintained.

4) $S G$ Constraints: The power output, acceptable ramp levels, and daily energy requirement for each SG $r \in \mathcal{R}$ is constrained by:

$$
\begin{array}{lr}
0 \leq P_{r h} \leq \bar{P}_{r h}, \quad 0 \leq Q_{r h} \leq \bar{Q}_{r h}, & \forall r, h \\
-r d_{r} \leq P_{r h}-P_{r(h-1)} \leq r u_{r}, & \forall r, h \\
\sum_{h \in \mathcal{H}} P_{r h} \leq e_{r} \cdot \bar{P}_{r}, & \forall r
\end{array}
$$

where $r d_{r}$ and $r u_{r}$ are the ramp-up and ramp-down limits while $e_{r}$ is the daily capacity factor of the SG.

5) CBG Constraints: Currently, grid codes have been updated to allow for CBGs operation at power factor other than 1 to allow for voltage support. The limitations on power output of each CBGs $c \in \mathcal{C}$ are defined by:

$$
0 \leq P_{c h} \leq \bar{P}_{c h}, \quad 0 \leq Q_{c h} \leq \tan (\bar{\phi}) \cdot \bar{P}_{c h}, \quad \forall c, h,
$$

where $\cos \bar{\phi}$ is the maximum power factor of the CBG defined by the grid code.

6) Steady-state Voltage Security and Thermal Loading Constraints: The steady-state voltage at each node should remain within the normal operating range (Fig. 1-region A) bounded by limits $\underline{V}_{p r e}^{2} / \bar{V}_{p r e}^{2}$ equivalent to $\underline{v} / \bar{v}$ defined as:

$$
\underline{v} \leq v_{n h} \leq \bar{v}, \quad v_{h \mid n=\mathrm{pcc}}=1, \quad \forall n, h
$$

while the line thermal loading limit $\bar{S}_{n n^{d}}$ is ensured by:

$$
P_{n n^{d} h}^{2}+Q_{n n^{d} h}^{2} \leq \bar{S}_{n n^{d}}^{2}, \quad \forall\left(n, n^{d}\right) \in \mathcal{T}, h
$$

where $\mathcal{T}$ is a set of lines connecting neighbouring nodes.

7) Dynamic Voltage Security Constraints: The response of the MG in the event of a fault-triggered islanding is dictated by the behaviour of loads, generators, and network, described by a model of differential-algebraic equations. In addition, the transient response may trigger changes in the controls (such as DVS for CBGs and over/under excitation for SGs) defined by discrete states. The resulting system model is a set of Differential-Algebraic-Discrete (DAD) equations. If we want to constrain the dynamic response of the voltage to ensure it does not violate the technical limits defined in Fig. 1, a dynamically constrained optimization problem should be formulated. This includes $\boldsymbol{f}(\dot{\boldsymbol{x}}, \boldsymbol{x}, \boldsymbol{y}, \boldsymbol{z})=\mathbf{0}$ describing the system dynamics and $\boldsymbol{h}(\boldsymbol{x}, \boldsymbol{y}, \boldsymbol{z}) \leq \mathbf{0}$ describing the path constraints (e.g., the voltage trajectory limits displayed in Fig. 1). $\boldsymbol{x}$ are differential states and $\boldsymbol{y}$ are the algebraic states respectively, while $z$ are discrete control variables.

To solve the dynamically constrained optimization problem, several methods have been proposed in literature [18]. The most common approach involves the discretization of the DAD equations (using an explicit or implicit integration formula) into static algebraic equations that are then embedded in the optimization problem [19], [20]. These constraints would take the form:

$$
\begin{gathered}
\boldsymbol{f}\left(\boldsymbol{x}_{\tau+1}, \boldsymbol{x}_{\tau}, \boldsymbol{y}_{\tau}, \boldsymbol{z}_{\tau}\right)=0 \\
\boldsymbol{h}\left(\boldsymbol{x}_{\tau+1}, \boldsymbol{x}_{\tau}, \boldsymbol{y}_{\tau}, \boldsymbol{z}_{\tau}\right) \leq \mathbf{0} \\
\underline{\boldsymbol{z}} \leq \boldsymbol{z}_{\tau} \leq \overline{\boldsymbol{z}}, \quad \underline{\boldsymbol{x}} \leq \boldsymbol{x}_{\tau} \leq \overline{\boldsymbol{x}}
\end{gathered}
$$

where $\tau$ is the time step used when algebraizing the DADs.

However, this approach introduces a huge computational burden. Each step of the discretization introduces a set of optimization states with inter-temporal coupling between them. For instance, embedding a small system with 200 DADs, discretized over a $2 \mathrm{~s}$ simulation horizon at a $10 \mathrm{~ms}$ time-step, would introduce a minimum of 40000 new optimization states! Further, assuming a multi-period problem (as the one in this work) where the constraint needs to be applied at each hour, then one can easily see that the problem becomes intractable. 


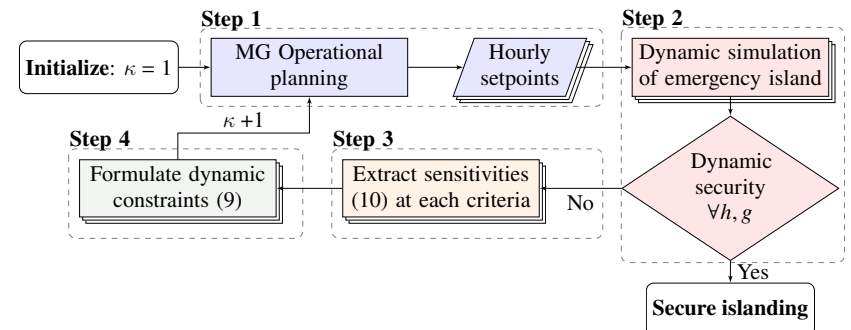

Fig. 2. Proposed solution algorithm.

To reduce the computational burden, in this study, we employ a direct sequential approach [18]. That is, we include in the optimization problem only the linearized equations involved with the voltage path constraints related to Fig. 1 instead of the full system defined in (8). Thus, the number of equations reduces significantly to just three (one for each of the path constraints). However, using the linearized path constraints instead of the full system (8) necessitates to resort to an iterative process for updating the constraint parameters. Therefore, at each iteration $k$ of this process, the constraints take the form:

$$
\begin{gathered}
\Delta V_{k+1}^{\mathrm{cr}}=\delta_{V_{k}^{\text {cr }}}^{P_{k}^{\text {grid }}} \cdot \Delta P_{k+1}^{\text {grid }}+\delta_{V_{k}^{\text {cr }}}^{Q_{\text {rid }}^{\text {grid }}} \cdot \Delta Q_{k+1}^{\text {grid }} \\
V_{k+1}^{\mathrm{LVRT}} \geq V_{\text {min }}, \quad V_{k+1}^{\mathrm{HVRT}} \leq V_{\max } \\
\underline{V}_{\text {rec }} \leq V_{k+1}^{\mathrm{rec}} \leq \bar{V}_{\mathrm{rec}}
\end{gathered}
$$

where superscript $\mathrm{cr} \in\{\mathrm{LVRT}, \mathrm{HVRT}, \mathrm{rec}\}$ correspond to the voltage magnitudes at LVRT, HVRT and post fault recovery, respectively, and $\delta_{a}^{b}$ is the sensitivity of $a$ to changes in $b$. $\Delta V_{k+1}^{\mathrm{cr}}=V_{k+1}^{\mathrm{cr}}-V_{k}^{\mathrm{cr}}$ denotes the change in controlled state $V_{k+1}^{\mathrm{cr}}$ from modifying the active and reactive power exchanged between MG and main grid by $\Delta P_{k+1}^{\text {grid }}$ and $\Delta Q_{k+1}^{\text {grid }}$, respectively. Constraints (9b) and (9c) are applied to ensure operation within technical limits (see the boundaries of regions B, C, and D in Fig. 1), between times $t_{0}$ to $t_{c}$ for $\mathrm{cr} \in\{\mathrm{LVRT}, \mathrm{HVRT}\}$ and $t_{r}$ for $\mathrm{cr} \in\{\mathrm{rec}\}$.

Given that the islanding constraints need to be satisfied at each hour $h$ of the problem, (9) is applied separately for each hour at each generator-coupled node. The final problem is therefore a mixed integer second-order cone programming problem that can be efficiently solved in polynomial time.

\section{B. Solution Algorithm}

Embedding constraints (9) into the optimization problem allows to perform preventive re-scheduling to ensure all operating points in the planning horizon adhere to the security requirements in the event of islanding. Therefore, we propose an iterative algorithm that couples the optimization problem with a time-domain simulation at each operating hour. This allows to determine if the system dynamics during the fault and just after islanding remain within the thresholds and to extract the path constraint sensitivities for the dynamic voltage criteria in (9). The tasks involved at each iteration $\kappa$ are outlined in Fig. 2 and detailed as follows:

Step 1: Solve the initial operational planning problem (Section III-A) for the entire planning horizon of the MG

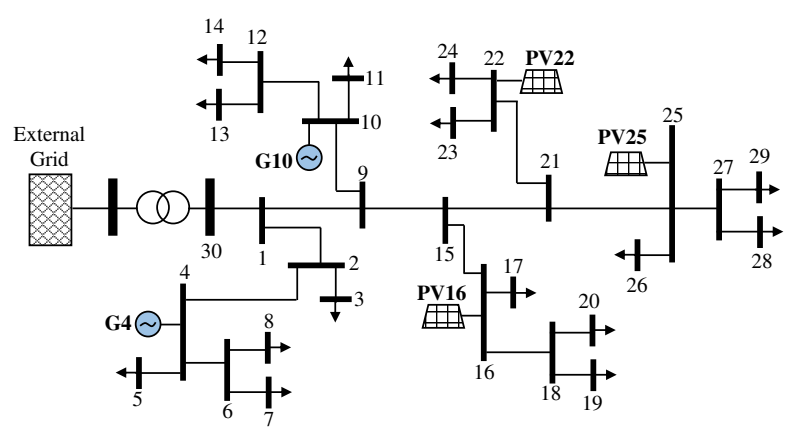

Fig. 3. One-line diagram of test system.

without constraints (9) to derive the hourly operating points of the grid-connected MG. Set $\kappa=1$.

Step 2: For each operating point derived at Step 1, simulate an emergency islanding of the MG and check that the FRT and transient recovery voltage trajectories at each generator node do not violate the security limits.

Step 3: If any operating point results in security violations in Step 2, extract the sensitivity coefficients $\delta_{a}^{b}$ for each of the voltage security criteria in (9). The sensitivities are approximated using a finite differences approach with a small variation in the active/reactive power exchange:

$$
\begin{aligned}
\delta_{V_{\kappa h}^{\mathrm{cr}}}^{[P / Q]_{\kappa h}^{\mathrm{grid}}} & =\frac{\partial V_{\kappa h}^{\mathrm{cr}}}{\partial[P / Q]_{\kappa h}^{\mathrm{grid}}} \\
& =\frac{V_{\kappa h}^{\mathrm{cr}}\left([P / Q]_{\kappa h}^{0+}\right)-V_{\kappa h}^{\mathrm{cr}}\left([P / Q]_{\kappa h}^{0-}\right)}{2 \Delta[P / Q]}
\end{aligned}
$$

where $[P / Q]_{\kappa h}^{0+}=[P / Q]_{\kappa h}^{0}+\Delta[P / Q]$ and $[P / Q]_{\kappa h}^{0-}=$ $[P / Q]_{\kappa h}^{0}-\Delta[P / Q]$, with $[P / Q]_{\kappa h}^{0}$ the initial power exchange computed in Step 1. That is, $[P / Q]_{\kappa h}^{0+}$ (resp. $[P / Q]_{\kappa h}^{0-}$ ) implies an increase (resp. decrease) in power flow "from" the MG "to" the main grid.

Step 4: Apply the dynamic security constraints indicated in (9) to the operational planning problem in Step 1 for each hour and re-solve.

\section{CAse Study Results}

\section{A. System Description}

The 30-bus, $20 \mathrm{kV}$, distribution network shown in Fig. 3 was used to test the proposed algorithm. The network topology and line parameters were obtained from [21]. The network feeds 15 loads modeled as constant power loads in the optimization problem, while voltage sensitive loads and induction machines at buses 14, 20 and 28 are considered for the dynamic simulation. The photovoltaic (PV) units (CBGs) are modeled as detailed in [16], while for the SGs a $6^{\text {th }}$-order model equipped with the DEGOV1 speed governor and the IEEE AC1A exciter is adopted. The peak load consumption is 13 MVA with a total capacity of 8.4 MVA for the 3 PV units while the 2 SGs have a combined rating of 14 MVA. Fig. 4 shows the load and PV generation profiles adopted from [22] in Texas during 2016. The active power import, export, and SG production costs were set to $15 \$ / \mathrm{MW}, 5 \$ / \mathrm{MW}$ and 40 $\$ / \mathrm{MW}$, respectively, with reactive power costs considered at $10 \%$ of the corresponding active power cost. 


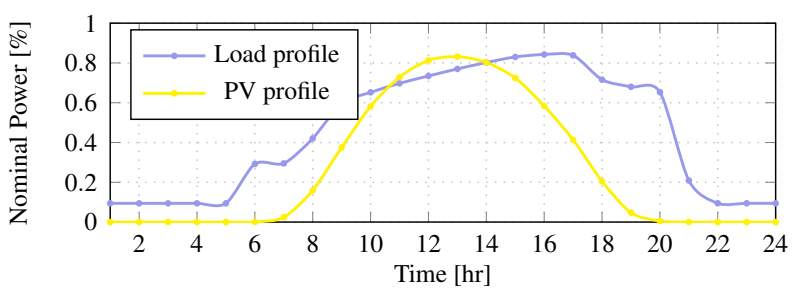

Fig. 4. Load and PV power generation patterns.

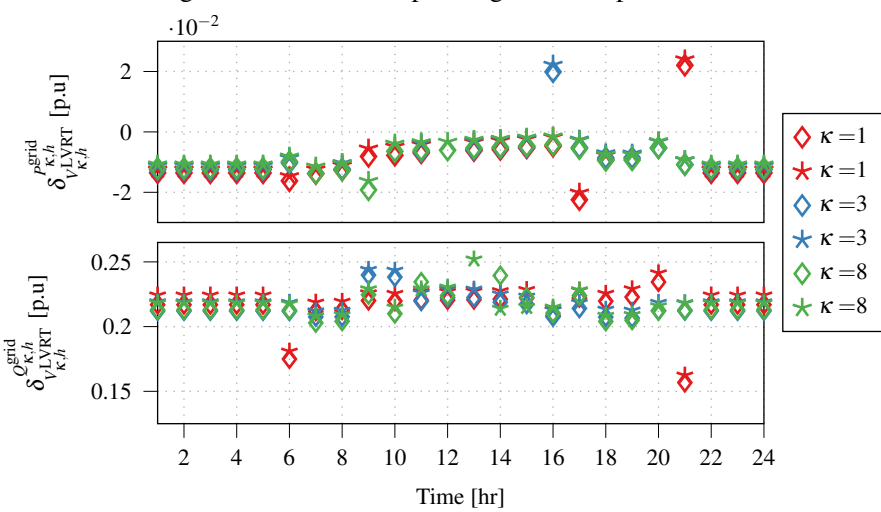

Fig. 5. Sensitivity coefficients of LVRT voltage to active and reactive power exchange at the main grid for SG G4 $(\diamond)$ and CBG PV16 $(\star)$ coupled nodes.

The dynamic simulation was performed with PyRAMSES [23], while the optimization model was implemented in Pyomo [24] and Gurobi [25] employed as a solver.

From Fig. 1, the LVRT and HVRT limits are set at $V_{\min }=0.45$ p.u. and $V_{\max }=1.2$ p.u. (region C and D, respectively) while the post-fault voltage recovery limits are set to $\underline{V}_{\text {rec }}=0.9$ p.u. and $\bar{V}_{\text {rec }}=1.1$ p.u. (region B). The steady-state voltages in normal operation are set within bounds of $\underline{V}_{\text {pre }}=0.95$ p.u. to $\bar{V}_{\text {pre }}=1.05$ p.u. (region A). The dynamic evolution of voltage during emergency islanding is simulated after a three phase fault at the high-voltage bus occuring at $t_{0}=1 \mathrm{~s}$ with $t_{c}$ and $t_{r}$ set at $0.1 \mathrm{~s}$ and $2 \mathrm{~s}$ after the fault occurrence. The MG performs an emergency islanding after five cycles by opening the interconnection at the PCC (node 30).

\section{B. Simulation Results}

Fig. 5 shows the hourly sensitivity coefficients denoting the effect of the MG dynamic voltage security criterion to variations in power exchanged with the main grid at different iterations and for the two types of generating units. The initial and final iterations of the algorithm are depicted with $\kappa=1$ and $\kappa=8$. At each hour, the level of power imbalance in the MG in case of islanding depends on the operating point dictated by the load and PV profiles (Fig. 4). It can be seen that overall the effect of active power variation remains minimal and in some instances inverse to that of reactive power.

Moreover, Fig. 5 shows that an increase in the active power imbalance, i.e. an increase in $\mathrm{MG}$ active power import, results in a higher voltage nadir at FRT with an average $1.2 \%$ increase for both types of units. On the other hand, a decrease in reactive power imbalance, i.e. a decrease in $\mathrm{MG}$ reactive power import, will improve the voltage nadir at FRT by an average of $24 \%$. While this effect is true for the majority of the operating
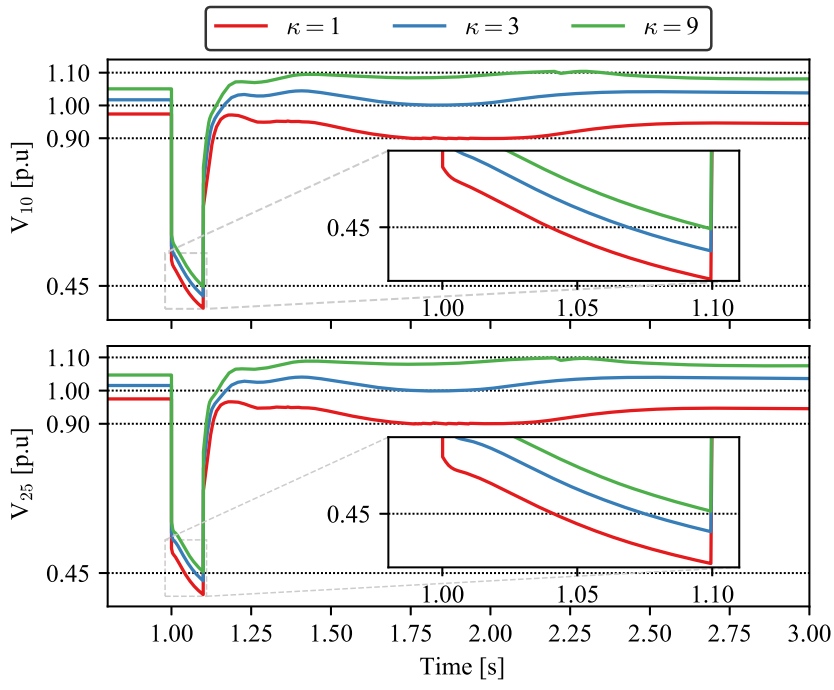

Fig. 6. Voltage evolution at nodes 10 and 25 at hour-16 operating point for different iterations.

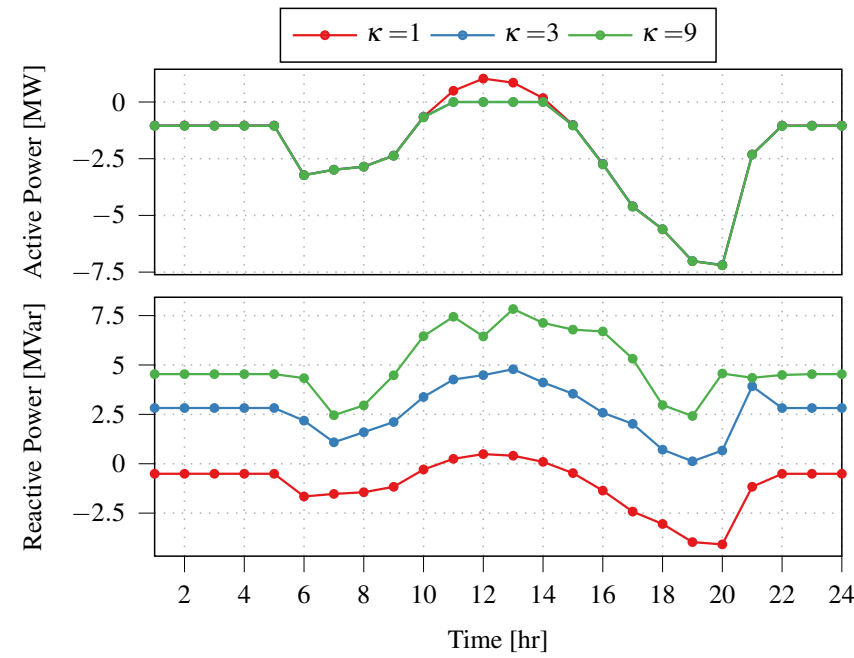

Fig. 7. Comparison of active and reactive power exchange with the grid for different iteration stages.

conditions, Fig. 5 indicates outlier lower sensitivities at hours 6 and and 21. This is usually the effect of SG and CBG controls getting limited during the transient response. As expected, a decrease in both active and reactive power imports prior to islanding improves the dynamic performance of the MG.

Fig. 6 depicts the voltage evolution in the case of an islanding event at hour 16 for the SG and CBG connected at nodes 10 and 25, respectively. At iterations greater than $\kappa=1$, the dynamic constraints are updated reducing the violations in LVRT voltage levels. However, this is dependent on the level of both active and reactive power reserves available in the MG. Nevertheless, it can be clearly seen that the algorithm achieves to bring the voltages within the desired range.

Fig. 7 shows the preventive re-schedules of active and reactive power over different iterations required to meet the dynamic voltage criterion. As expected, the algorithm makes heavy use of the cheaper and with higher sensitivity local reactive power generation to achieve the goal. Active power is only used during peak hours. While the CBGs (PV units) will prioritize active power in the steady-state, the SGs units 


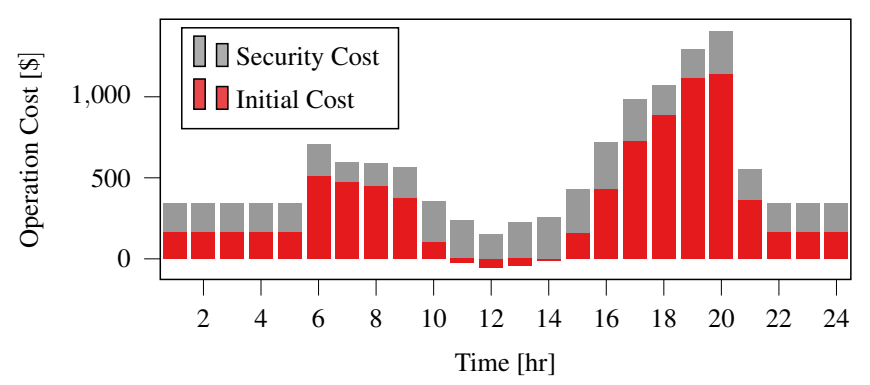

Fig. 8. Total costs incurred in ensuring voltage security at each islanding hour (negative values denote profit).

increase their reactive power production leading to a boost in steady-state voltages as depicted in Fig. 6. Increasing local reactive power generation results in lower reactive power reserves during the emergency periods. Nevertheless, during the fault conditions, reactive power priority provided by the CBGs results in improved dynamic voltage support. The two-stage combined support, i.e. increasing steady-state performance in addition to the DVS capability of the converters, allows for improved dynamic voltage security as is visualized in Fig. 6.

Finally, Fig. 8 indicates the initial optimal cost without voltage security constraints and the required cost increase to ensure survivability of the MG (final iteration). It can be derived that a cost increment of $53 \%$ on the total operation cost is required to ensure system survivability and security on islanding. As expected, there is a cost associated to securing and making the system more resilient to islanding transients.

\section{CONClusion}

MG islanding is an effective way to enhance resilience when the bulk transmission grid is in an emergency situation. However, analysing only the post-islanding steady-state conditions is not enough to ensure the survivability of the MG during the islanding transients. In this work, we propose a methodology to ensure that the transient voltage response of the MG during islanding, as well as the post-islanding steady-state, are within the secure limits. Instead of incorporating the full DAD model describing the transients in the optimization problem, we only include linear constraints to provide a computationally efficient solution, and we update them through an iterative algorithm. The performance of the proposed technique was demonstrated on a medium-voltage network with results indicating that rescheduling of both active and reactive powers can be adopted to enhance system resilience. In the future, the algorithm will be extended to incorporate the effect of power variations at individual generator nodes, thus allowing a more costeffective re-scheduling. Moreover, the impact of uncertainties will be analysed as well as the interactions between voltage and frequency transient dynamics.

\section{REFERENCES}

[1] M. Panteli, D. N. Trakas, P. Mancarella, and N. D. Hatziargyriou, "Boosting the power grid resilience to extreme weather events using defensive islanding," IEEE Trans. on Smart Grid, vol. 7, no. 6, pp. 2913-2922, 2016.

[2] A. Hussain, V.-H. Bui, and H.-M. Kim, "Microgrids as a resilience resource and strategies used by microgrids for enhancing resilience," Applied Energy, vol. 240, pp. 56 - 72, 2019.
[3] S. Eftekharnejad, V. Vittal, G. T. Heydt, B. Keel, and J. Loehr, "Impact of increased penetration of photovoltaic generation on power systems," IEEE Trans. on Power Sys., vol. 28, no. 2, pp. 893-901, 2013.

[4] A. H. Kasem Alaboudy, H. H. Zeineldin, and J. Kirtley, "Microgrid stability characterization subsequent to fault-triggered islanding incidents," IEEE Trans. on Power Delivery, vol. 27, no. 2, pp. 658-669, 2012.

[5] W. Zheng, P. Crossley, B. Xu, and H. Qi, "Transient stability of a distribution subsystem during fault-initiated switching to islanded operation," Int. Journ. of Elec. Pow. \& Energy Sys., vol. 97, pp. 418 - 427, 2018.

[6] "IEEE Standard for Interconnecting Distributed Resources with Electric Power Systems," IEEE Std 1547-2003, pp. 1-28, 2003.

[7] "The Technical Basis for the New WECC Voltage Ride-Though (VRT) Standard," A White Paper Developed by the Wind Generation Task Force, Tech. Rep., June 2007.

[8] G. Lammert, T. Heß, M. Schmidt, P. Schegner, and M. Braun, "Dynamic grid support in low voltage grids - fault ride-through and reactive power/voltage support during grid disturbances," in 2014 Power Systems Computation Conf., 2014, pp. 1-7.

[9] M. Islam, N. Mithulananthan, and M. J. Hossain, "Dynamic voltage support by tl-pv systems to mitigate short-term voltage instability in residential dn," IEEE Trans. on Power Sys., vol. 33, no. 4, pp. 43604370, 2018.

[10] K. Kawabe, Y. Ota, A. Yokoyama, and K. Tanaka, "Novel dynamic voltage support capability of photovoltaic systems for improvement of short-term voltage stability in power systems," IEEE Trans. on Power Sys., vol. 32, no. 3, pp. 1796-1804, 2017.

[11] H. Liu, V. Krishnan, J. D. McCalley, and A. Chowdhury, "Optimal planning of static and dynamic reactive power resources," IET Gen., Transn. Dist., vol. 8, no. 12, pp. 1916-1927, 2014.

[12] G. Hou and V. Vittal, "Trajectory sensitivity based preventive control of voltage instability considering load uncertainties," IEEE Trans. on Power Sys., vol. 27, no. 4, pp. 2280-2288, 2012.

[13] F. Capitanescu and T. Van Cutsem, "Preventive control of voltage security margins: a multicontingency sensitivity-based approach," IEEE Trans. on Power Sys., vol. 17, no. 2, pp. 358-364, 2002.

[14] L. Jin, R. Kumar, and N. Elia, "Security constrained emergency voltage stabilization: A model predictive control based approach," in 2008 47th IEEE Conf. on Decision and Control, 2008, pp. 2469-2474.

[15] M. Paramasivam, A. Salloum, V. Ajjarapu, V. Vittal, N. B. Bhatt, and S. Liu, "Dynamic optimization based reactive power planning to mitigate slow voltage recovery and short term voltage instability," IEEE Trans. on Power Sys., vol. 28, no. 4, pp. 3865-3873, 2013.

[16] G. Chaspierre, P. Panciatici, and T. Van Cutsem, "Dynamic equivalent of a distribution grid hosting dispersed photovoltaic units," in Proc. 10th Bulk Power Syst. Dyn. \& Control Symp. (IREP), 2017, pp. 1-12.

[17] M. Farivar and S. H. Low, "Branch flow model: Relaxations and convexification-part i," IEEE Trans. on Power Sys., vol. 28, no. 3, pp. 2554-2564, 2013.

[18] L. T. Biegler and I. E. Grossmann, "Retrospective on optimization," Computers \& Chemical Engin., vol. 28, no. 8, pp. 1169 - 1192, 2004.

[19] M. La Scala, M. Trovato, and C. Antonelli, "On-line dynamic preventive control: an algorithm for transient security dispatch," IEEE Trans. on Power Sys., vol. 13, no. 2, pp. 601-610, 1998.

[20] S. Abhyankar, G. Geng, M. Anitescu, X. Wang, and V. Dinavahi, "Solution techniques for transient stability-constrained optimal power flow - part i," IET Gen., Trans. Dist., vol. 11, no. 12, pp. 3177-3185, 2017.

[21] E. Kägi-Kolisnyc, "Distribution Management System Including Dispersed Generation and Storage in a Liberalized Market Environment," Ph.D. dissertation, EPFL, Lausanne, Switzerland, 2009.

[22] N. Blair, N. DiOrio, J. Freeman, P. Gilman, S. Janzou, T. Neises, and M. Wagner, "System Advisor Model (SAM) General Description (Version 2017.9.5)," National Renewable Energy Laboratory, Golden, CO, Tech. Rep. NREL/ TP-6A20-70414, May 2018.

[23] P. Aristidou, S. Lebeau, and T. Van Cutsem, "Power system dynamic simulations using a parallel two-level schur-complement decomposition," IEEE Trans. on Power Sys., vol. 31, no. 5, pp. 3984-3995, 2016.

[24] W. E. Hart, J.-P. Watson, and D. L. Woodruff, "Pyomo: modeling and solving mathematical programs in python," Mathematical Programming Computation, vol. 3, no. 3, pp. 219-260, 2011.

[25] Gurobi Optimization, LLC, "Gurobi optimizer reference manual," 2020. [Online]. Available: http://www.gurobi.com 